\title{
ON THE NONUNIQUE EQUILIBRIUM STATES OF A SHALLOW ARCH SUBJECTED TO A UNIFORM LATERAL LOAD*
}

\author{
BY \\ ARNOLD D. KERR AND LOTFI EL-BAYOUMY \\ New York University
}

Summary. Using the exact solutiom of a shallow elastic arch clamped at both ends and subjected to a uniform lateral load, it is shown that, in addition to the equilibrium states usually discussed in the literature, also other equilibrium states, which correspond to higher modes of deformation, do exist. It is shown that there exist only a finite number of equilibrium branches which correspond to these higher modes, the number depending upon the shallowness of the arch. A number of graphs are presented which demonstrate these findings. The paper concludes with the proof that all states of equilibrium which correspond to the branches of higher order are unstable.

Introduction. The elastic shallow arch or infinitely long shallow panel, clamped or simply supported, and subjected to a uniform lateral load as shown in Fig. 1, was analyzed in the past by a number of investigators [1]-[\$]. Whereas in references [1], [3], [5] and [6] approximate methods were used to solve the resulting nonlinear formulations, in references [2], [4], [7] and [8] the resulting two nonlinear differential equations were reduced to a linear one, which is amenable to an exact analysis. Recently, during the preparation of reference [\$], it became apparent that the obtained exact solution contains, in addition to the usual equilibrium states shown in Fig. 2, also other equilibrium branches. A similar finding, in connection with the buckling of spherical shells, was reported recently in [9]. The purpose of the present paper is to determine, by means of the exact solution derived in [S], the additional equilibrium branches and the corresponding stresses and deformations of the shallow clamped arch, and then discuss their stability.

The equilibrium branches. With the usual assumptions for thin shallow arches and the notation of Fig. 1, the principle of stationary total potential energy yields the two simultaneous nonlinear differential equations $[8,10]$ :

$$
E A \hat{\epsilon}_{\theta \theta}+\frac{1}{R}\left(A E \hat{\epsilon}_{\theta \theta} \hat{u}_{, \theta}\right)_{, \theta}-\left(\frac{E I}{R} \hat{\kappa}\right)_{. \theta \theta}+p_{0} R=0, \quad\left(E A \hat{\epsilon}_{\theta \theta}\right)_{, \theta}=0,
$$

with the boundary conditions for the clamped ends

$$
\hat{u}\left( \pm \theta_{0}\right)=0 ; \quad \hat{u}_{, \theta}\left( \pm \theta_{0}\right)=0 ; \quad \hat{v}\left( \pm \theta_{0}\right)=0
$$

where the ()-terms refer to the axis of the arch, $E$ is Young's modulus, $I$ is the moment

* Received Scptember 24, 1969. This research was sponsored by the Air Force Office of Scientific Research, Office of Aerospace Research, United States Air Force under AFOSR Grant No. AF-AFOSR67-0813. 


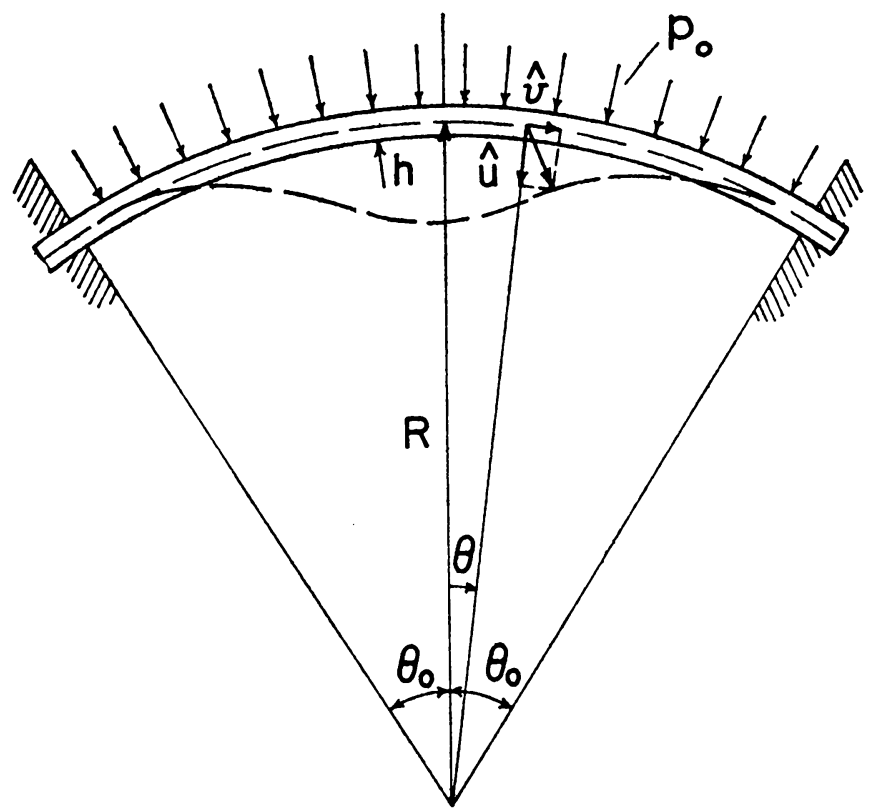

FIG. 1

of inertia, $A$ is the area of cross section and

$$
\hat{\epsilon}_{\theta \theta}=\frac{1}{R}\left(\hat{v}_{, \theta}-\hat{u}\right)+\frac{1}{2 R^{2}} \hat{u}_{, \theta}^{2}, \quad \hat{\kappa}=\frac{1}{R^{2}} \hat{u}_{, \theta \theta} .
$$

It may be shown (for details the reader is referred to [8] or [10]) that the exact solution of the above nonlinear problem for symmetrical deformations is

$$
\hat{u}_{s}(\theta)=h K\left[\bar{p}_{0} \frac{K}{\left(\lambda \theta_{0}\right)^{2}}-1\right]\left\{\frac{\cos (\lambda \theta)-\cos \left(\lambda \theta_{0}\right)}{\left(\lambda \theta_{0}\right) \sin \left(\lambda \theta_{0}\right)}-\frac{1}{2}\left[1-\left(\frac{\lambda \theta}{\lambda \theta_{0}}\right)^{2}\right]\right\},
$$

in conjunction with the equation

$$
\begin{aligned}
& \bar{p}_{0}^{2} \frac{K^{2}}{\mu^{4}}\left(4-\frac{5}{3} \mu^{2}-3 \mu \operatorname{ctg} \mu\right.\left.-\mu^{2} \operatorname{ctg}^{2} \mu\right) \\
&-2 \bar{p}_{0} \frac{K}{\mu^{2}}\left(2-\mu^{2}-\mu \operatorname{ctg} \mu-\mu^{2} \operatorname{ctg}^{2} \mu\right) \\
& \quad+\left(\mu \operatorname{ctg} \mu-\mu^{2} \operatorname{ctg}^{2} \mu-\frac{1}{3} \mu^{2}-\frac{\mu^{4}}{3 K^{2}}\right)=0
\end{aligned}
$$

where

$$
\mu=\lambda \theta_{0} ; \quad \lambda^{2}=T_{0} R^{2} /(E I) ; \quad \bar{p}_{0}=p_{0} R^{2} h /(E I) ; \quad K=R \theta_{0}^{2} / h .
$$

The term $T_{0}$ is the axial compression force in the arch. In view of the second equation in (1) it follows that

$$
T_{0}=-E A \hat{\epsilon}_{\theta \theta}=\text { const } \quad \text { in }-\theta_{0} \leqq \theta \leqq \theta_{0} .
$$

Eq. (4) and Eq. (5) were numerically evaluated for $K=6,8,10,12,15$ by determining 
for each $K$ value and a set of $\mu$-values the corresponding values of $\bar{p}_{0}$ from Eq. (5), and then by computing for each obtained pair of $\mu$ and $\bar{p}_{0}$ the corresponding value $\hat{u}_{s}(\theta) / h$ from Eq. (4). The results for $\hat{u}_{s}(o)$ are presented, as solid lines, in Figs. 2 to 6.

Each figure consists of two parts. The upper part contains the load displacement graph, $\hat{u}(o) / h$ versus $\bar{p}_{0}$, and the lower part contains the $\left(\lambda \theta_{0}\right)$ versus $\bar{p}_{0}$ graph, $\left(\lambda \theta_{0}\right)$ being related to the axial force $T_{0}$ as shown in (6). The solid lines represent the equilibrium branches of the symmetrical deformations.

It may be seen that for $K=6$ there is only one symmetrical equilibrium branch

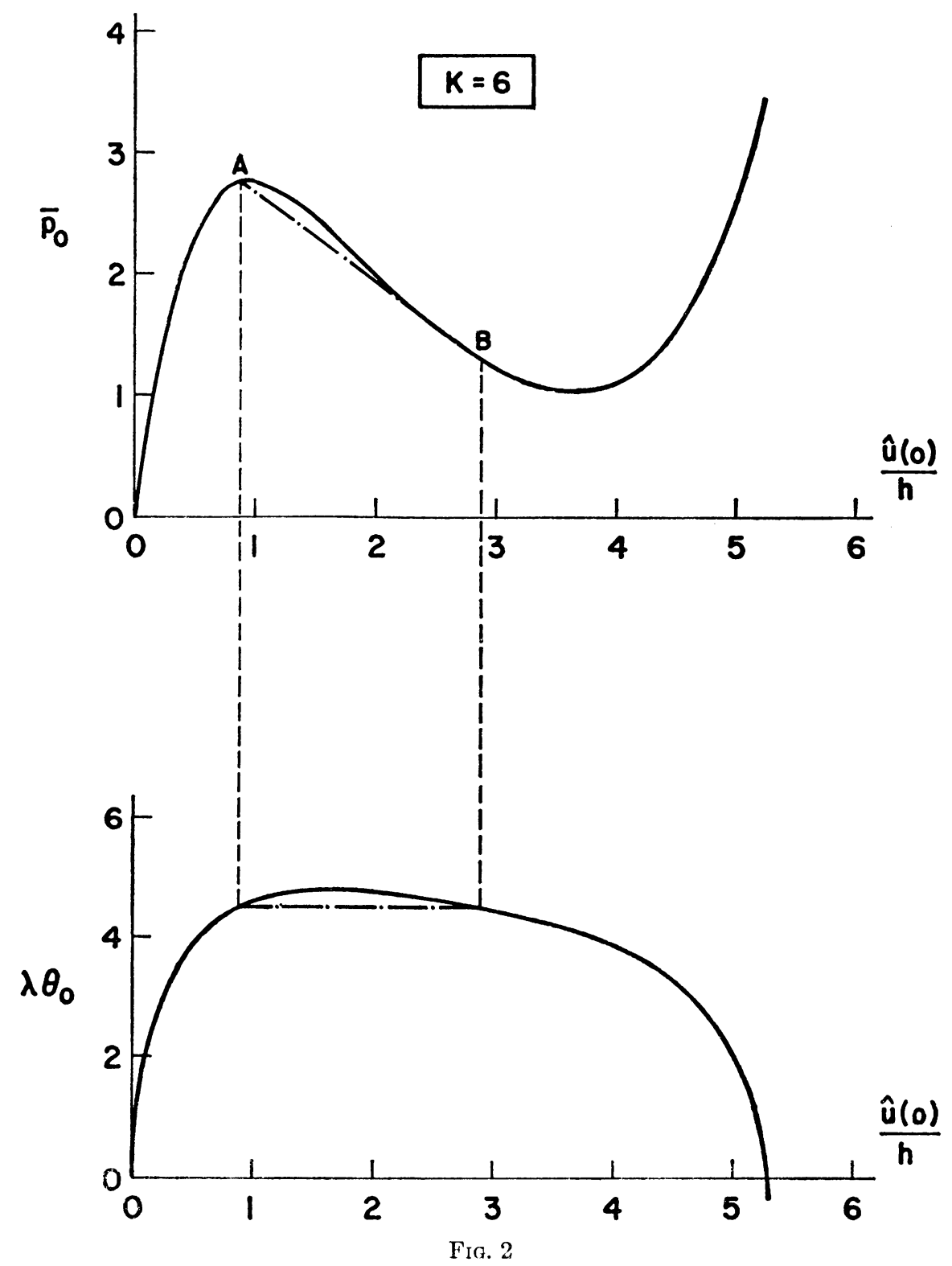




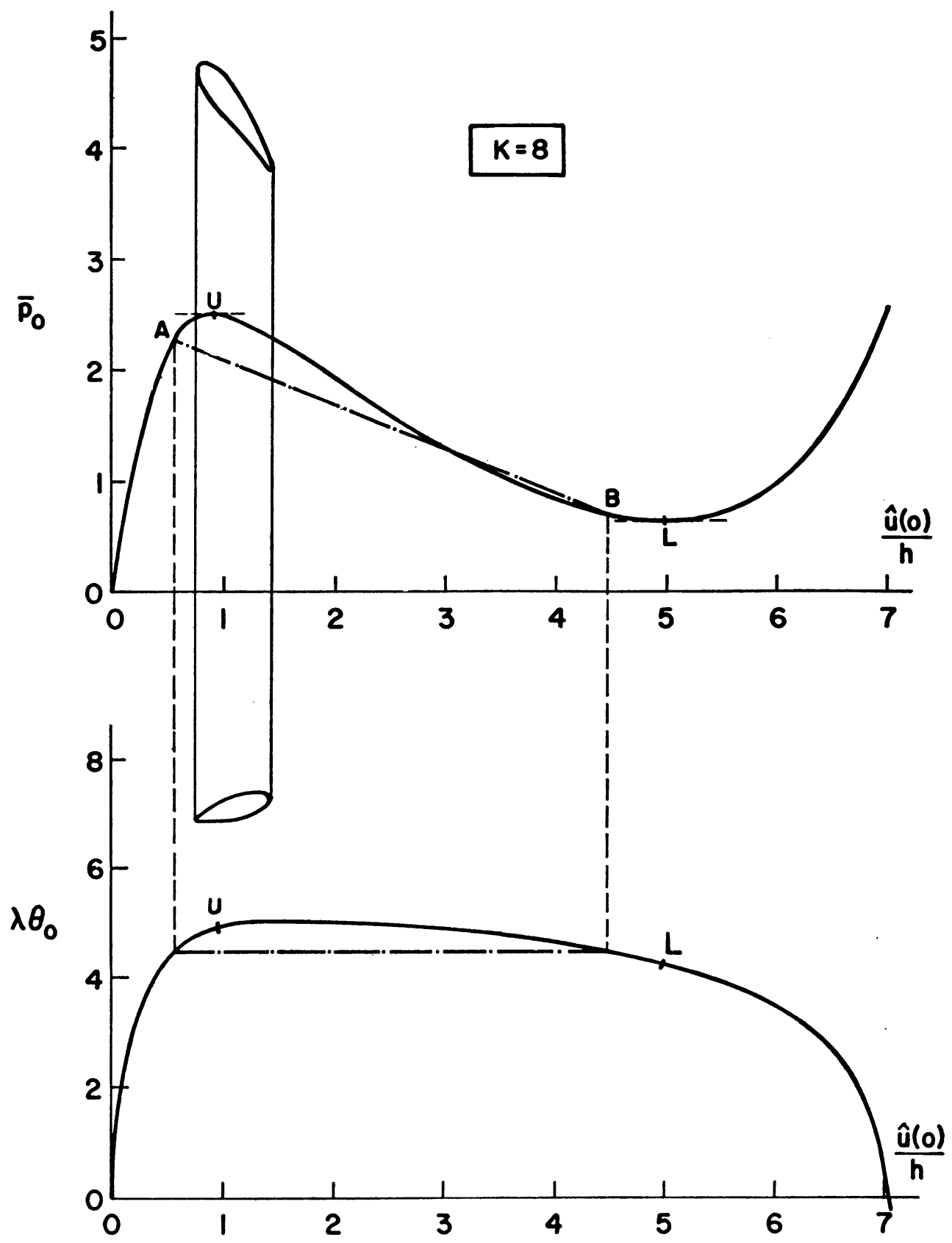

FIG. 3 


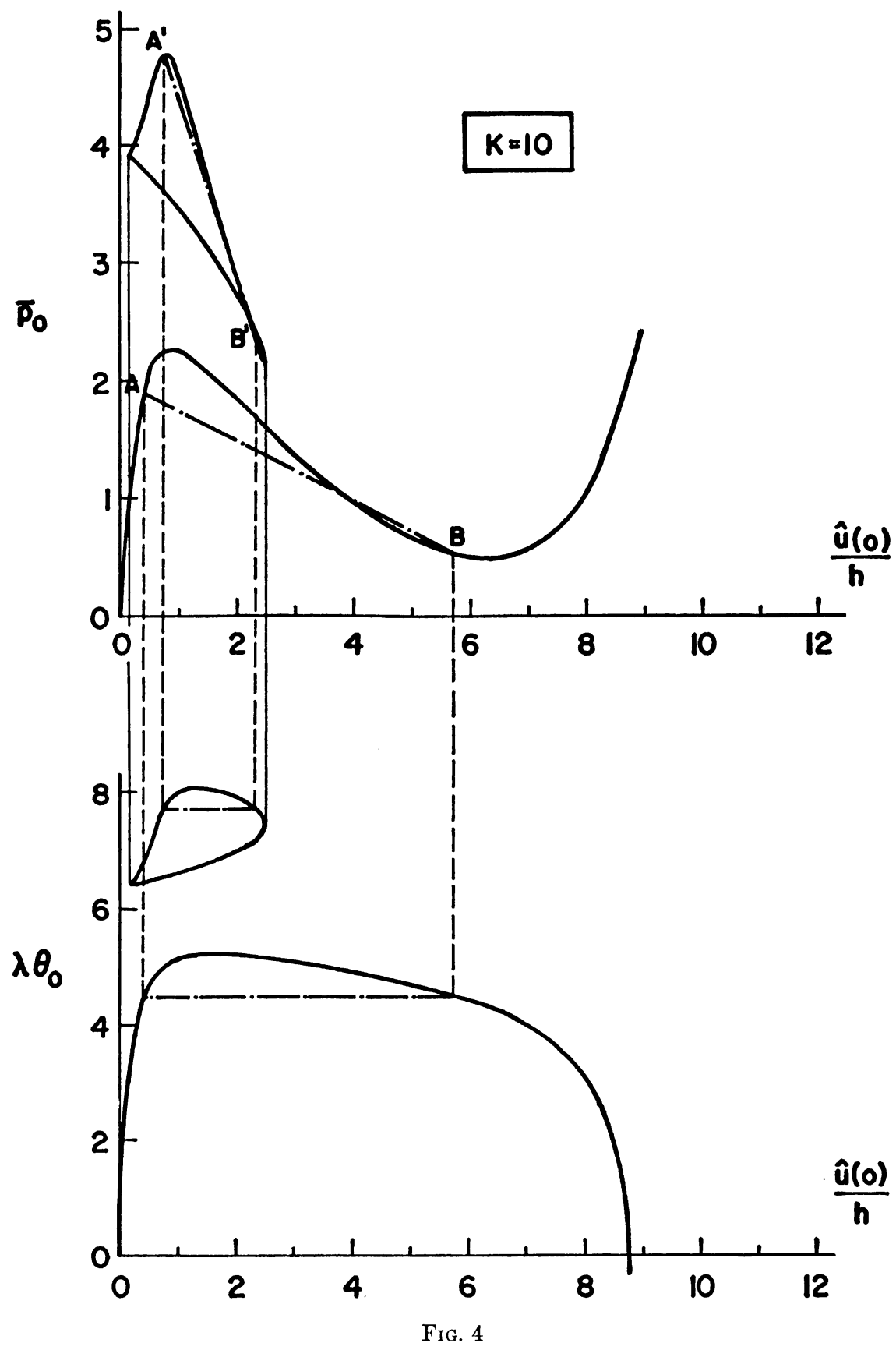




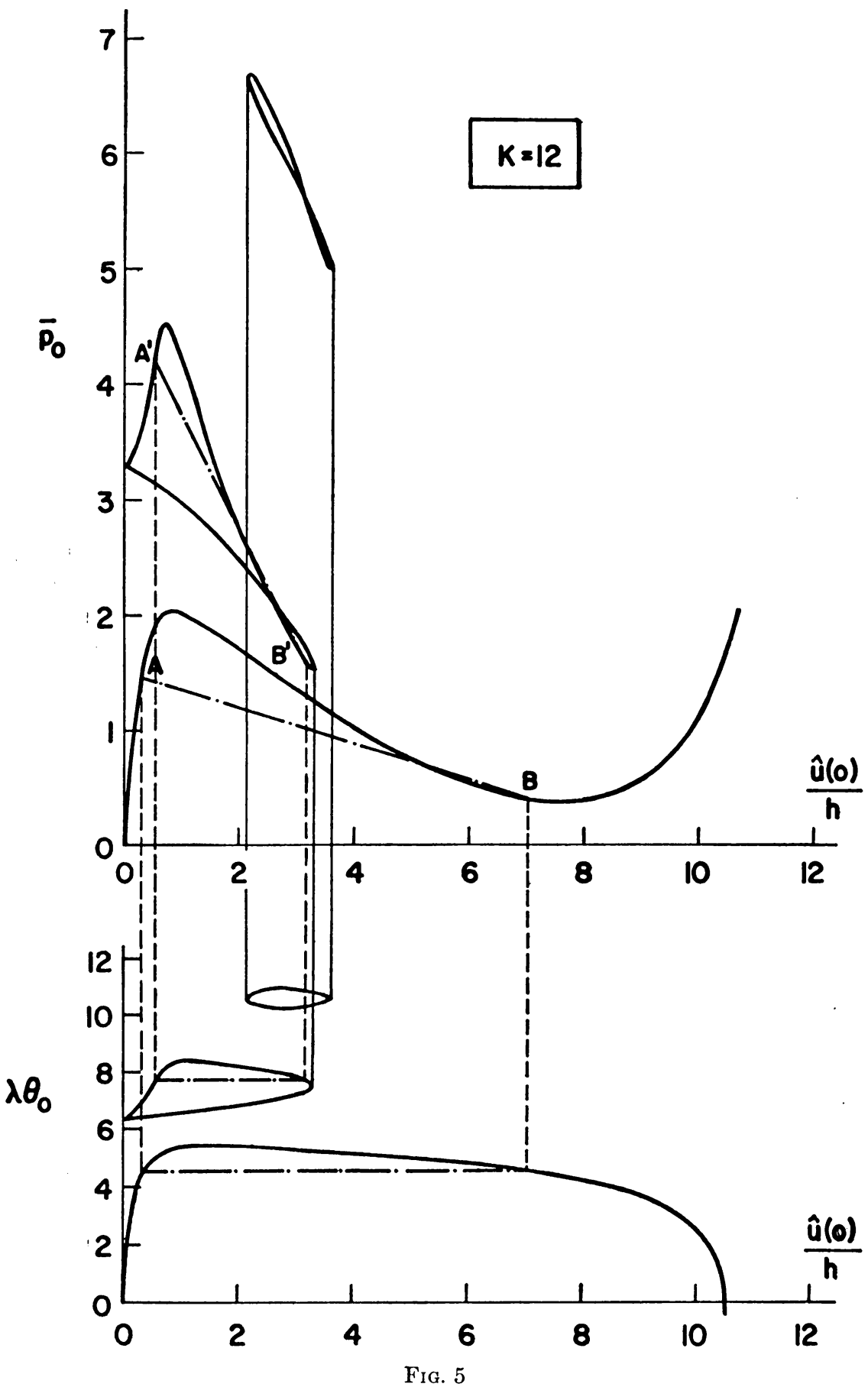




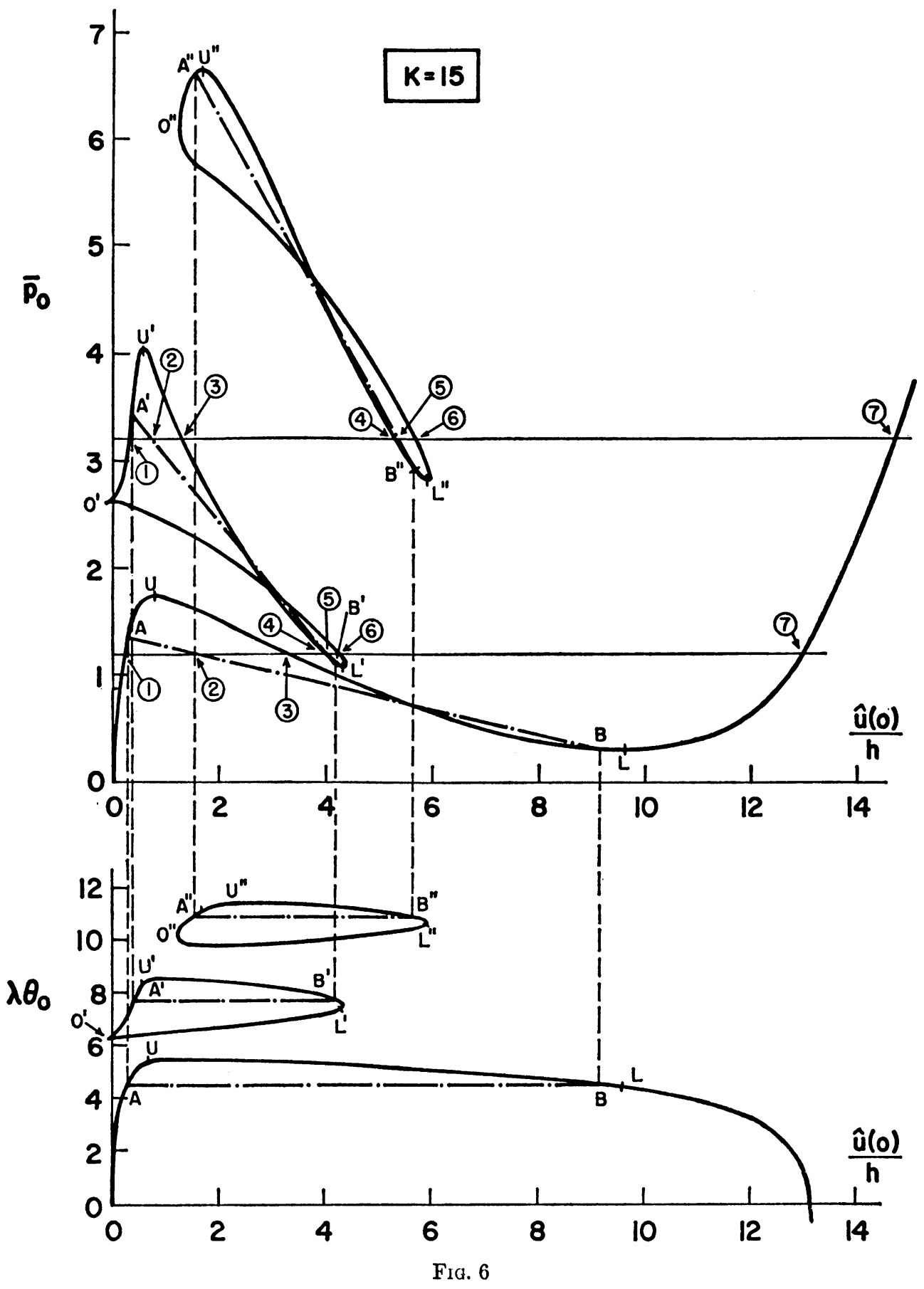


of the type presented in references [1]-[8]. Figs. 3 to 6 show, however, that with increasing $K$, in addition to the main equilibrium branch there exist also other equilibrium branches which form closed loops. These additional branches do not emanate from the main branch and do not pass through the origin of the coordinate system. These figures also show, that the number of these additional branches increases with increasing $K$.

It may also be seen from the figures that the axial force $T_{0}$ is higher for the additional branches than for the corresponding main equilibrium branch, which indicates that the additional equilibrium branches correspond to higher modes of deformation. This is confirmed by checking the displacement graphs shown in Fig. 7 and Fig. 8 .

There is a basic difference between the main branch and the additional equilibrium branches. Whereas the main branch is obtained in an experiment by continuously varying the load starting from zero (see description of experiment in [6]), the additional equilibrium branches may be realized by prebending the elastic arch to a certain form (see Fig. 7 or Fig. 8) and then applying the corresponding pressure at which the arch will be in equilibrium (not necessarily stable, as will be shown later).

It may be also shown ([8] and [10]) that nonsymmetric deformations may take place when $\operatorname{tg}\left(\lambda \theta_{0}\right)=\left(\lambda \theta_{0}\right)$. This equation is satisfied for an infinite number of roots

$$
\left(\lambda^{*} \theta_{0}\right)=4.4934,7.7253, \cdots .
$$

It is found that for a given shallow arch and any one of the roots (which corresponds to a real situation) the compression force $T_{0}$ is not only constant throughout the arch but it remains constant throughout the nonsymmetrical deformations. It is equal to the value at the corresponding bifurcation point and is denoted by $T_{0}^{*}$. Thus, the

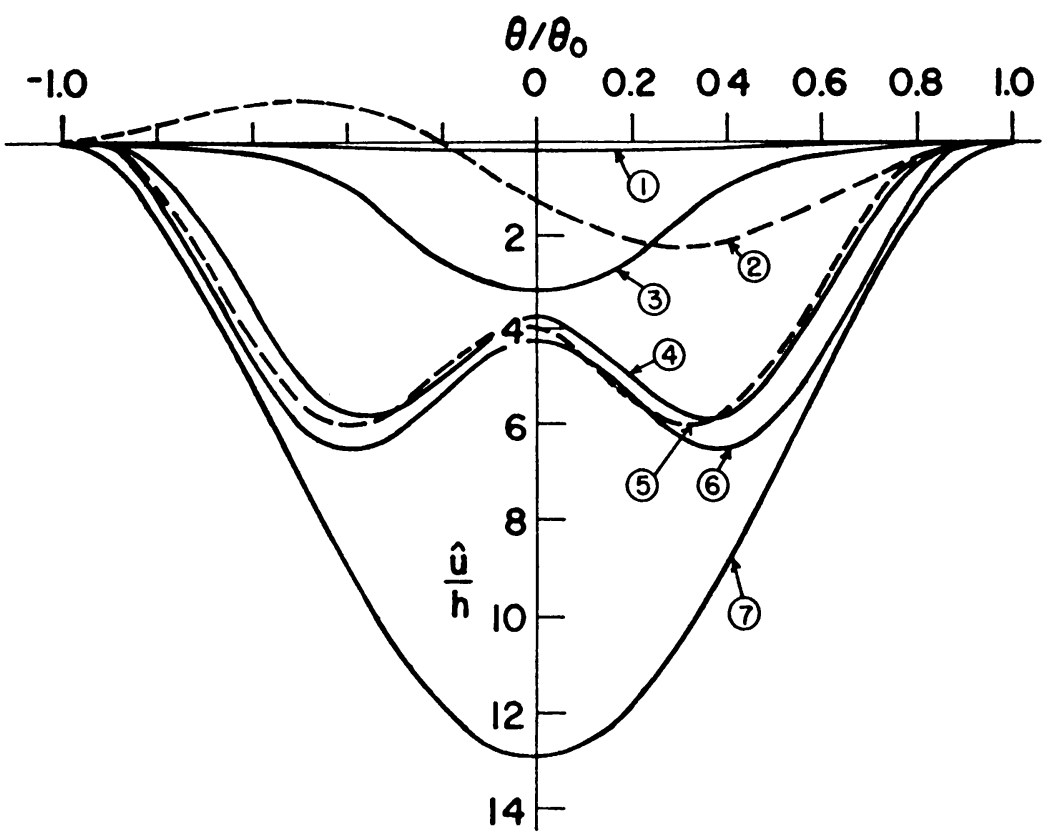

FIG. $7 \hat{u}$-profiles for $K=15$ and $\bar{p}_{0}=1.2$ 


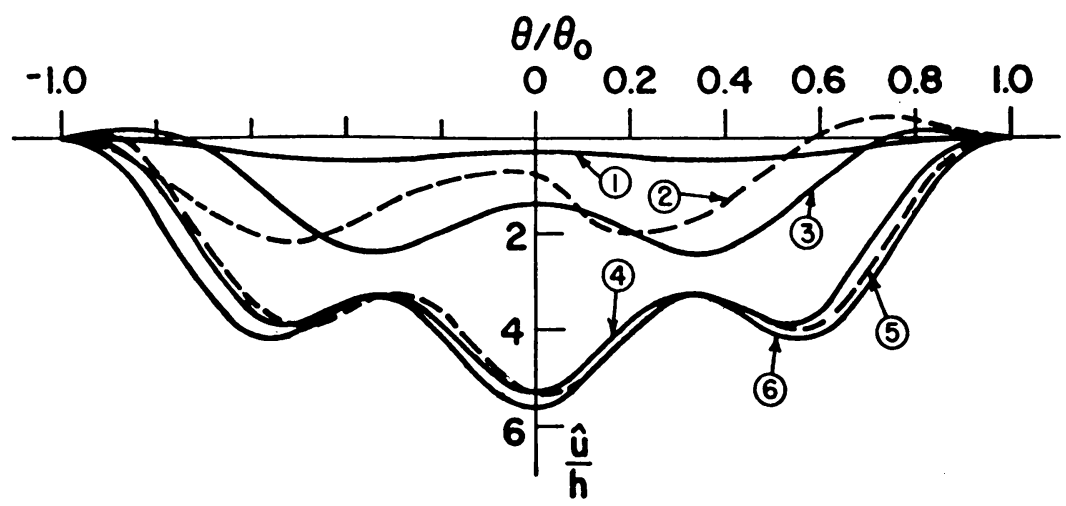

Fig. 8. $\mathfrak{a}$-profiles for $K=15$ and $\tilde{p}_{0}=3.2$

bifurcation pressures, $\bar{p}_{b}$, are obtained by substituting the equation $\operatorname{tg}\left(\lambda^{*} \theta_{0}\right)=\left(\lambda^{*} \theta_{0}\right)$ into Eq. (5). The obtained pressures are

$$
\bar{p}_{1,2}=\frac{\left(\lambda^{*} \theta_{0}\right)^{2}}{5 K}\left[3 \pm 2\left(1-\frac{5}{4} \frac{\left(\lambda^{*} \theta_{0}\right)^{2}}{K^{2}}\right)^{1 / 2}\right] .
$$

The $\bar{p}_{1}$ values are the bifurcation pressures at the points $A, A^{\prime}, A^{\prime \prime}, \ldots$ whereas the $\bar{p}_{\mathbf{2}}$ values are the pressures at the points $B, B^{\prime}, B^{\prime \prime}, \ldots$ From Eq. (9) it follows that bifurcation points will exist for

$$
K>(\sqrt{ } 5 / 2)\left(\lambda^{*} \theta_{0}\right) .
$$

Substituting the roots given in (8) into condition (10), it is found that for $K=6$ and $K=8$ only the first root yields real values for the bifurcation pressures, for $K=10$ and $K=12$ only the first two roots yield real pressures, for $K=15$ only the first three roots yield real pressures, etc. Comparing this finding with the $\bar{p}_{0}$ versus $\hat{u}(o) / h$ graphs shown in Figs. 2 to 6 , it may be seen that not all higher equilibrium branches contain bifurcation points and also how the occurrence of these points depends upon the parameter $K$. For a fixed $\left(\lambda^{*} \theta_{0}\right)$, the expression for nonsymmetrical displacements $\hat{u}(o)$ reduces to the equation of a straight line, shown in the upper parts of Figs. 2 to 6 as dash-dot lines. The corresponding $\left(\lambda \theta_{0}\right)$ graphs are shown, as dash-dot lines, in the lower parts of of these figures.

The stability of the new equilibrium branches. In the previous section the equilibrium states of the shallow arch were discussed without establishing which of them are stable and which are not. In this section we determine the stability of the new equilibrium branches.

The following analysis is based on the criterion that for an equilibrium configuration to be stable the corresponding $\Pi$ has to be a minimum with respect to the $\Pi$ 's of all kinematically admissible neighboring states (which do not have to be states of equilibrium). For a recent discussion on the subject see [11].

As uning the kinematically admissible perturbed displacements in the form

$$
\hat{u}(\theta)+\alpha \tilde{u}(\theta) ; \quad \hat{v}(\theta)+\alpha \tilde{v}(\theta)
$$

where $\hat{u}$ and $\hat{v}$ are displacements which correspond to any state on the equilibrium 
branches, $\tilde{u}$ and $\tilde{v}$ are kinematically admissible perturbations, and $\alpha$ is a small parameter, it follows, since for all determined equilibrium branches $\delta \Pi=0$, that when

$$
\begin{array}{ll}
\delta^{2} \Pi>0, & \text { system is stable, } \\
\delta^{2} \Pi<0, & \text { system is unstable }
\end{array}
$$

provided that $\delta^{2} \Pi$ is the dominant term in the expansion of $\Delta \Pi$. When $\delta^{2} \Pi=0$, the behavior of variations of higher order have to be studied, although usually the condition $\delta^{2} \Pi=0$ yields for a stable branch the transition condition at which the state of equilibrium ceases to be stable.

For the problem under consideration

$$
\mathrm{II}=\int_{-\theta_{0}}^{+\theta_{0}}\left[\frac{E R}{2}\left(A \hat{\epsilon}_{\theta \theta}^{2}+I \hat{\kappa}^{2}\right)-p_{0} R \hat{u}\right] d \theta .
$$

Noting (6), (7) and the second equation in (3), $\Pi$ becomes

$$
\Pi=\int_{-\theta_{0}}^{+\theta_{0}}\left\{\frac{E I}{2 R^{4}}\left[\frac{\left(\lambda \theta_{0}\right)^{4}}{12 K^{2}}+\hat{u}_{. \theta \theta}^{2}\right]-p_{0} \hat{u}\right\} R d \theta .
$$

Thus

$$
\delta^{2} \Pi=\left[\frac{\partial^{2} \Pi[(\tilde{)}+\alpha \tilde{()}]}{\partial \alpha^{2}}\right]_{\alpha=0}=\int_{-\theta_{0}}^{+\theta_{0}}\left(E A R \tilde{\epsilon}_{\theta \theta}^{2}+\frac{E I}{R^{3}} \tilde{u}_{. \theta \theta}^{2}+\frac{E A}{R} \hat{\epsilon}_{\theta \theta} \tilde{u}_{\cdot \theta}^{2}\right) d \theta
$$

where

$$
\tilde{\epsilon}_{\theta \theta}=\frac{1}{R}\left(\tilde{v}_{. \theta}-\tilde{u}\right)+\frac{1}{R^{2}} \hat{u}_{. \theta} \tilde{u}_{, \theta} .
$$

It should be noted that in $\delta^{2} \Pi$ the load parameter appears implicitly in the first and third term. Noting (7), Eq. (15) becomes

$$
\delta^{2} \Pi=\int_{-\theta_{0}}^{+\theta_{0}}\left(E A R \tilde{\epsilon}_{\theta \theta}^{2}+\frac{E I}{R^{3}} \tilde{u}_{. \theta \theta}^{2}-\frac{T_{0}}{R} \tilde{u}_{. \theta}^{2}\right) d \theta
$$

where $T_{0}$ is the compression force in the arch for a corresponding equilibrium state, $E A R>0$, and $E I / R^{3}>0$.

The stability discussions of the main branch available in the literature suggest the possibility that the additional branches, which correspond to symmetric as well as to nonsymmetric deformations, are unstable. To prove instability of an equilibrium configuration is relatively simple, since it is usually sufficient to show that $\delta^{2} \Pi<0$ for any chosen, kinematically admissible, $\tilde{u}$ and $\tilde{v}$. In view of (11) and (2), $\tilde{u}$ and $\tilde{v}$ have to satisfy the boundary conditions

$$
\begin{aligned}
\tilde{u}\left( \pm \theta_{0}\right) & =0, \\
\tilde{u}, \theta\left( \pm \theta_{0}\right) & =0, \\
\tilde{v}\left( \pm \theta_{0}\right) & =0 .
\end{aligned}
$$

For this purpose we choose [7]

$$
\tilde{u}(\theta)=B\left[\left(\lambda_{1}^{*} \theta\right) \cos \left(\lambda_{1}^{*} \theta_{0}\right)-\sin \left(\lambda_{1}^{*} \theta\right)\right]
$$


where $B$ is an arbitrary constant and $\lambda_{1}^{*} \theta$ is the lowest root given in (8). The proof is simplified by using the Jacobi form of $\delta^{2} \Pi$ and by noting that (21) satisfies the eigenvalue problem of neutral equilibrium for which $\delta^{2} \Pi=0$. We thus obtain

$$
\delta^{2} \Pi=-\left(\lambda_{1}^{*^{2}}-\lambda^{2}\right) \frac{E I}{R^{3}} \int_{-\theta_{0}}^{+\theta_{0}} \tilde{u}_{, \theta \theta} \tilde{u} d \theta .
$$

Substituting (21) into (22) yields

$$
\delta^{2} \Pi=\frac{B^{2} E I \lambda_{1}^{*}\left(\lambda_{1}^{*} \theta_{0}\right)^{3}}{R^{3}\left[1+\left(\lambda_{1}^{*} \theta_{0}\right)^{2}\right]}\left(\lambda_{1}^{* 2}-\lambda^{2}\right) .
$$

For all equilibrium branches, except the main branch, $\lambda>\lambda_{1}^{*}$. Therefore, for these branches

$$
\delta^{2} \Pi<0 .
$$

Substituting (21) into $\delta^{2} \Pi$ and $\delta^{4} \Pi$, the remaining terms in the expansion of $\Delta \Pi$, it may be shown that $\delta^{2} \Pi$ is the dominant term. Hence, all equilibrium states which correspond to the additional branches shown in Figs. 3 and 6 are unstable.

\section{References}

[1] S. Timoshenko, Buckling of flat curved bars and slightly curved panels, J. Appl. Mech. 2, A17-A20 (1935)

[2] K. O. Friedrichs and J. J. Stoker, Seminar on nonlinear elasticity, Section IX, New York University, $1947 / 48$

[3] Y. C. Fung and A. Kaplan, Buckling of low arches and curved beams of small curvature, Technical Note 2840, NACA (Currently NASA) Washington, D. C., 1952.

[4] M. S. Kornishin and Kh. M. Mushtari, The instability of an infinitely long shallow cylindrical strip subjected to a normal uniform pressure, Izv. Kazansk. Fil. Akad. Nauk USSR Ser. Fiz.-Mat. Tehn. Nauk 7, 36-50 (1955) (Russian) See also: Nonlinear theory of thin elastic shells, Chapter XI, by Kh. M. Mushtari and K. Z. Galimov, Tatknigoizdat, Kazan, 1957; English transl., NASA-T'-62, 1961.

[5] A. S. Volmir, Flexible plates and shells. Chapter VI, GITTL, Moscow, 1956 (Russian)

[6] A. Gelsvik and S. Bodner, Energy criterion and snap buckling of arches, Proc. ASCE, Journal of the Engineering Mechanics Division, EM5, Oct. 1962, pp. 87-134.

[7] L. H. Schreyer and E. F Masur, Buckling of shallow arches, Proc. ASCE, Journal of the Engineering Mechanics Division, EM4, August 1966, pp. 1-19

[8] A. D. Kerr and M. T. Soifer, The linearization of the prebuckling state and its effect on the determined buckling loads, J. Appl. Mech. Dec. 1969, 775-783

[9] H. B. Keller and A. W. Wolfe, On the nonunique equilibrium states and buckling mechanism of spherical shells, J. Soc. Indust. Appl. Math. 13, 674-705 (1965)

[10] A. D. Kerr and L. El-Bayoumy, Equilibrium and stability of a shallow arch subjected to a uniform lateral load, New York University Report No. AA-68-44, November 1968

[11] W. T. Koiter, The energy criterion of stability for continuous elastic bodies, I, II, Nederl. Akad. Wetensch. Proc. Ser. B 68, 178-202 (1965) 